\title{
Case-control study of endoscopic polypectomy in clinic (EPIC) versus endoscopic sinus surgery for chronic rhinosinusitis with polyps*
}

\author{
Shaun J. Kilty',2, Andrea Lasso², Leandra Mfuna-Endam³, Martin Y. Desrosiers ${ }^{3}$ \\ 2The Ottawa Hospital Research Institute (OHRI), Ottawa, Canada \\ 3 Department of Otolaryngology, Hôtel-Dieu Hospital, Centre de Recherche du Centre Hospitalier de I'Université de Montréal \\ (CRCHUM), Montreal, Canada
}

Rhinology 56; 2: 155-157, 2018

https://doi.org/10.4193/Rhin17.115

*Received for publication:

May 29, 2017

Accepted: December 13, 2017

\begin{abstract}
Introduction: Endoscopic Polypectomy In Clinic (EPIC) is a recently described deescalated form of endoscopic sinus surgery (ESS) performed in the outpatient clinic for patients with chronic rhinosinusitis with polyps (CRSwNP). The quality of life benefit of EPIC in comparison to ESS is not known. The purpose of this study was to determine if the disease specific quality of life measured with the SNOT-22 attained with EPIC is similar to that attained with ESS for patients with CRSwNP.
\end{abstract}

Methods: A multi-institutional observational case-control study was performed to evaluate quality of life improvement in patients treated with ESS and EPIC for CRSwNP with a 3 month follow-up. Predicted probability of undergoing EPIC was calculated by fitting a logistic regression model using clinically relevant variables. EPIC patients were matched to ESS patients in a 1:1 fashion.

Results: 24 pairs were analyzed after matching. There was no statistical difference in the post-treatment SNOT-22 scores or proportion of patients achieving a minimal clinically important difference.

Conclusions: In appropriate CRSwNP patients, the EPIC procedure may provide disease specific quality of life improvement similar to that seen with patients who undergo traditional ESS.

Key words: Chronic sinusitis, nasal polyps, endoscopic sinus surgery, polypectomy

\section{Introduction}

Endoscopic Polypectomy In Clinic (EPIC) is a recently developed, deescalated form of endoscopic sinus surgery (ESS) performed in the Outpatient Clinic instead of the Operating Room for patients with chronic rhinosinusitis with polyps and the primary impairments of nasal obstruction and olfactory impairment. EPIC appears to provide quality of life improvement equivalent to that reported for ESS ${ }^{(1,2)}$. In the US, the total direct cost of illness for CRS care has been estimated at over 60 billion USD annually, nearly $1 \%$ of the US healthcare budget in $2011^{\text {(3) }}$. EPIC has a health system cost that is nearly one-tenth of ESS and a pilot cost-effectiveness study has demonstrated the favorability of EPIC over ESS (4). Polyp recurrence following ESS is common, with $35 \%$ of patients having polyp recurrence by 6 months and a need for a second surgery within 5 years for $20 \%$ of patients who receive surgical treatment ${ }^{(5,6)}$.

To date, all studies evaluating EPIC have used a single cohort treatment population. In order to better understand the clinical effects of EPIC in comparison to ESS, a study design utilizing a direct comparator ESS treatment group would be beneficial. The purpose of this study was to determine if EPIC is as effective as ESS in terms of quality of life improvement in patients who have chronic rhinosinusitis with polyps.

\section{Materials and methods}

A multi-institutional observational case-control cohort study was performed to evaluate quality of life improvement in patients receiving ESS and EPIC as surgical treatments for CRS with nasal polyps (CRSwNP). The study was carried out utilizing data collected at two University-based sinus centers under the appro- 
val of each insititution's research ethics board. Patients receiving EPIC were part of an observational study where data on pre- and post-operative measures of symptom severity were collected using the SNOT-22 questionnaire. All EPIC patients completed a post-operative assessment at 3 months after the procedure. Patients who underwent ESS were part of an observational study in which data on symptom severity was collected using the SNOT-22 questionnaire. All patients completed a post-operative assessment, also 3 months after ESS.

EPIC is a procedure utilised for patients with CRSwNP whose primary complaint is nasal obstruction with minimal facial pain. Therefore the predicted probability of undergoing EPIC was calculated by fitting a logistic regression model, using the clinically relevant variables age, nasal obstruction and facial pain scores from the baseline SNOT-22. EPIC patients were matched to ESS patients on the propensity score in a 1:1 fashion with nearest neighbor matching without replacement using calipers of width equal to 0.2 of the standard deviation of the propensity score ${ }^{(7)}$.

Group comparisons after matching were carried out using paired t-test for continuous variables and McNemar's test for binary variables. A 2-tailed probability of less than 0.05 denoted the presence of a statistically significant difference. All statistical analyses were performed with Stata (version 12.1; StataCorp LP, College Station, USA).

\section{Results}

32 EPIC patients and 59 ESS patients were available for analysis. The characteristics of the patients in the unmatched and matched samples are displayed in Table 1. Eight patients in the EPIC group were dropped because they were outside of common support. After matching, the absolute standardized difference of means was $<25 \%$ and the variance ratio was 1.45 on the matched variables indicating adequate balance ${ }^{(8)}$.

Twenty-four pairs were analyzed after matching (Table 2). The post-operative SNOT-22 score of those who underwent ESS was $21.87(S D=22.05)$ and the post-operative SNOT-22 score of those who underwent EPIC was $13.79(S D=9.25)$. There was no statistical or clinical significant difference in the post-treatment SNOT22 scores when comparing the EPIC and ESS groups $(p=0.09)$. Eighteen (75\%) of the patients who had ESS achieved minimal clinically important difference (MCID) while 22 (91\%) who had EPIC achieved MCID. The proportion of patients achieving MCID did not differ between EPIC and ESS groups $(p=0.21)$.

\section{Discussion}

Endoscopic sinus surgery is the standard of treatment for patients with chronic rhinosinusitis with polyps who have failed medical treatment ${ }^{(9)}$. For a subgroup of patients with CRSwNP, the EPIC procedure has been shown to provide an improvement
Table 1. Characteristics of the unmatched sample.

\begin{tabular}{|lccc|}
\multicolumn{1}{|c}{ Variable } & EPIC (n=32) & ESS $(\mathbf{n = 5 9 )}$ & $\mathbf{p}$ \\
\hline Age mean (SD) & $60.12(15.17)$ & $51.22(12.34)$ & 0.0032 \\
\hline Sex female $\mathrm{n}(\%)$ & $9(28.13)$ & $22(37.29)$ & 0.379 \\
\hline $\begin{array}{l}\text { Facial Pain mean (SD) } \\
\text { Nasal Obstruction }\end{array}$ & $0.75(0.84)$ & $2(1.78)$ & 0.0003 \\
\hline $\begin{array}{l}\text { mean (SD) } \\
\text { Baseline SNOT-22 score } \\
\text { mean (SD) }\end{array}$ & $4.93(1.29)$ & $3.54(1.44)$ & 0.199 \\
\hline $\begin{array}{l}\text { Asthma n (\%) } \\
\text { Previous surgery n (\%) }\end{array}$ & $11(34.45)$ & $45.86(20.06)$ & 0.681 \\
\hline Allergies n (\%) & $17(53.13)$ & $41(69.49)$ & 0.001 \\
\hline
\end{tabular}

Table 2. Characteristics of the matched sample.

\begin{tabular}{|c|c|c|c|}
\hline Variable & EPIC $(n=24)$ & ESS $(n=24)$ & $\mathbf{p}$ \\
\hline Age mean (SD) & $57.37(12.02)$ & $55.66(10.57)$ & 0.575 \\
\hline Sex female $n(\%)$ & $6(25.00)$ & $7(29.17)$ & 1.00 \\
\hline Facial Pain mean (SD) & $0.91(0.88)$ & $0.95(1.45)$ & 0.873 \\
\hline $\begin{array}{l}\text { Nasal Obstruction } \\
\text { mean (SD) }\end{array}$ & $3.62(1.34)$ & $3.54(1.44)$ & 0.839 \\
\hline $\begin{array}{l}\text { Baseline SNOT-22 score } \\
\text { mean (SD) }\end{array}$ & $43.83(17.84)$ & 40.79 (18.91) & 0.562 \\
\hline Asthma n (\%) & $8(33.33)$ & $16(66.67)$ & 0.021 \\
\hline Previous surgery n (\%) & $13(54.17)$ & $16(66.67)$ & 0.581 \\
\hline Allergies n (\%) & $13(54.17)$ & $20(83.33)$ & 0.039 \\
\hline
\end{tabular}

in patient disease related quality of life similar to that reported for ESS ${ }^{(1,2)}$. Further, EPIC has been found previously to have a substantial health system cost savings and favorable cost-effectiveness in comparison to ESS ${ }^{(4)}$. In this subgroup of patients with CRSwNP, undergoing the EPIC procedure for the treatment has been shown to have a high probability of achieving a minimal clinically important difference in disease specific quality of life of $90 \%$, mirroring the favorable results seen in patients who have undergone endoscopic sinus surgery ${ }^{(2)}$.

In this study, we have shown that patients who undergo the EPIC procedure for the treatment of chronic rhinosinusitis with polyps have an equal probability of achieving a minimal clinically important difference in their disease specific quality of life and that the change in quality of life as measured by the SNOT-22 is not different from those patients who undergo endoscopic sinus surgery. This suggests that when EPIC is used to treat patients with CRSwNP, they can expect to attain a similar improvement in their sinonasal symptomatology and quality of life as they would achieve by undergoing ESS. Further, if treated with EPIC, this 
improvement in quality of life can be achieved with substantial cost savings for the healthcare system, as shown from prior study ${ }^{(4)}$.

Despite that this case-control study has demonstrated results with EPIC that are similar to those obtained when patients undergo ESS, it is important to note that this study has limitations. First, the study does have a small sample size thereby affecting the level of accuracy of the results. Similarly, although data collection was prospectively performed, the methodology of this study is retrospective opening it to the criticisms of such study design. Further, in order to ascertain if a selection bias exists, a sufficiently powered randomised controlled trial would be the best study design in order to determine the similarity of effects amongst these two procedures. Finally, the primary outcome measure, the SNOT-22 score, was assessed as a short-term outcome at 3 months for each surgical group. Although three months appears to be short, the landmark study completed by Hopkins et al. (2009) with a cohort of over 3000 patients who had undergone endoscopic sinus surgery for chronic sinusitis and who were followed for 60 months demonstrated that the SNOT-22 scores at 3 months following surgery were not different from those measured at 12, 36 or 60 months ${ }^{(6)}$ and that this remains true even when data from patients who had had revision surgery during the follow up period was removed. That is, three months after treatment the SNOT-22 does not appear to change substantially with further follow-up. Therefore, the 3-month (90 day) follow-up SNOT-22 score may be of sufficient duration to assess this quality of life outcome and provide a useful estimate of the twelve, thirty-six and sixty month SNOT22 scores. Furthermore, from the large cohort of patients who underwent a polypectomy procedure under general anesthesia in the UK national study, the sinonasal quality of life outcomes at 36 months were not different from those of patients who had undergone ESS ${ }^{(12)}$. Despite these earlier results, a long-term study is needed to determine the enduring impact of both EPIC and ESS on sinonasal quality of life in this CRSwNP subgroup.

\section{Conclusion}

In select patients with chronic rhinosinusitis with polyps the EPIC procedure may provide disease specific quality of life improvements similar to that seen with patients who undergo endoscopic sinus surgery. Given the available data, further study of the effects of EPIC and ESS utilising a randomised clinical trial methodology in this patient subgroup is warranted.

\section{Authorship contribution}

SJK: Study design, data collection and analysis, manuscript development, review of final manuscript. AL: Data collection and analysis, manuscript development, review of final manuscript. LME: Data collection and analysis, manuscript development, review of final manuscript. MYD: Study design, data collection and analysis, manuscript development, review of final manuscript.

\section{Conflict of interest}

No conflict of interest.

\section{References}

1. Kilty SJ. In-clinic endoscopic polypectomy for chronic rhinosinusitis with polyps: a treatment strategy pilot study in ten adults. Clin Otolaryngol, 2015. 40(3): 281-284.

2. Caulley L, Lasso A, Rudmik L, Kilty SJ. Pre-Treatment SNOT-22 Score Predicts Response to Endoscopic Polypectomy in Clinic (EPIC) 2017; 42(3):732-734

3. Caulley L, Thavorn K, Rudmik L, Cameron C, Kilty SJ. Direct costs of adult chronic rhinosinusitis by using 4 methods of estimation: Results of the US Medical Expenditure Panel Survey. J Allergy Clin Immunol, 2015; 136(6):1517-1522.

4. Rudmik L, Smith KA, Kilty S. Endoscopic Polypectomy in the Clinic (EPIC): A Pilot Cost-Effectiveness Analysis. Clin Otolaryngol, 2016; 41(2):110-117.

5. DeConde AS, Mace JC, Levy JM, Rudmik L, Alt JA, Smith TL. Prevalence of polyp recurrence after endoscopic sinus surgery for chronic rhinosinusitis with nasal polyposis.
Laryngoscope, 2017; 127(3):550-555.

6. Hopkins, C., et al., Long-term outcomes from the English national comparative audit of surgery for nasal polyposis and chronic rhinosinusitis. Laryngoscope; 2009; 119(12): 2459-2465.

7. Austin PC. Optimal caliper widths for propensity-score matching when estimating differences in means and differences in proportions in observational studies. Pharm Stat, 2011. 10(2): 150-161.

8. Rubin DB. Using Propensity Scores to Help Design Observational Studies: Application to the Tobacco Litigation. Health Services and Outcomes Research Methodology. 2001; 2(3):169-188.

9. Kilty S. Canadian guidelines for rhinosinusitis: practical tools for the busy clinician. BMC Ear Nose Throat Disord, 2012. 12: 1.

10. Hopkins C, Browne JP, Slack R, et al. The national comparative audit of surgery for nasal polyposis and chronic rhinosinusitis. Clin Otolaryngol, 2006. 31(5): 390-398.
11. Browne JP, Hopkins C, Slack R, et al., Healthrelated quality of life after polypectomy with and without additional surgery. Laryngoscope, 2006. 116(2):297-302.

Dr. Shaun Kilty

Department of Otolaryngology

Head and Neck Surgery

Ottawa Hospital, Civic Campus

259-737 Parkdale Ave.

Ottawa, ON

Canada

Tel: +1 (613) 798-5555 xt. 18514

Fax: +1 (613) 729-2412

E-mail: kiltysj@gmail.com 